\title{
A survey on Sentimental Analysis algorithms and techniques
}

\author{
Prof. Dr. Mohamed El- Zeweidy \\ Higher Institute of Computers and Information Technology, Computer Depart. , El. Shorouk \\ Academy, Cairo, Egypt \\ Email: dr.mohamedelzweidy@sha.edu.eg \\ Aml Ibrahim Kamal \\ Higher Institute of Computers and Information Technology, Computer Depart. , El. Shorouk \\ Academy, Cairo, Egypt \\ Email: amal.ibrahim@sha.edu.eg
}

\begin{abstract}
Social web has been the lead of data in recent years. The data on social web simulates the customer reviews, comments, and opinions of people about products, opinion, events, etc. The automatic mining technique that is used to gather and analyze text and classify it into positive, negative and neutral is called Sentimental analysis using Natural language processing. The main objective of this paper is to give explain insights on SA algorithms and techniques.
\end{abstract}

Index Terms-Sentimental Analysis (SA), Natural language processing, Opinion Mining 


\section{Introduction}

$\mathrm{N}$ owadays opinions are a very important part of our lives, where the opinion is an essential part of analyzing user behaviour. Sentiments can be recognized as emotions, or as judgments, opinions or ideas prompted or colored by emotions or susceptibility or feelings [1]. In Computational Linguistics, the focus is on opinions and sentiments rather than on feelings. There are two types of textual information: facts and opinions information. While the facts are objective expressions about objects, features, entities, events and their characteristics, opinions are ordinarily subjective expressions that identify people's sentiments, views or feelings toward objects, entities, events and their characteristics [2].

Sentiment analysis (also called: sentiment mining, sentiment classification, opinion mining, subjectivity analysis, review mining or appraisal extraction, and in some cases polarity classification) can deal with the computational handling of subjective, sentiment, and opinion in the text [2]. It plans to realize the attitude or opinion of a writer with respect to a certain topic or goal. The attitude could reflect his/her opinion and evaluation, his/her effective situation (what are the feelings of the writer at the time of recording the opinion) or the purpose emotional communication (What is the effect which is situated on the reader when reading the opinion of the writer). Moreover, it should be noted that in this context 'subjective' does not mean that something is not true [3].

\section{I.1Sentiment Analysis (SA) \& Natural Language processing (NLP):}

Sentiment analysis, opinion mining and subjectivity analysis are interrelated areas of research which use various techniques taken from Natural Language Processing (NLP), Information Retrieval (IR), structured and unstructured Data Mining (DM). Major part of data available worldwide, being unstructured (such as text, speech, audio, video etc.), poses important research challenges. To deal with such unstructured text data, traditional 
methods of NLP i.e. information retrieval and information extraction came into existence [5]. In order to get a sense of the extracted text, numerous research efforts have been witnessed in recent years leading to automated SA, an extended NLP area of research [6]. Sentiment analysis is not a single problem; instead it is a multi-faceted problem [7]. Various steps are needed to perform opinion mining from given texts, since texts for opinion mining is coming from several resources in diverse format. Data acquisition and data preprocessing are most common subtasks required for text mining and SA.

\section{I.2Generic sentimental analysis system:}

The general architecture of a generic sentiment analysis system is shown in Figure (1) and is organized as follows:

1. The input to the system is a corpus of documents in any format (PDF, HTML, XML, and Word, among others).

2. The documents in this corpus are converted to text and are preprocessed using a variety of linguistic tools such as stemming, tokenization, part of speech tagging, entity extraction, and relation extraction.

3. The system may also utilize a set of lexicons and linguistic resources.

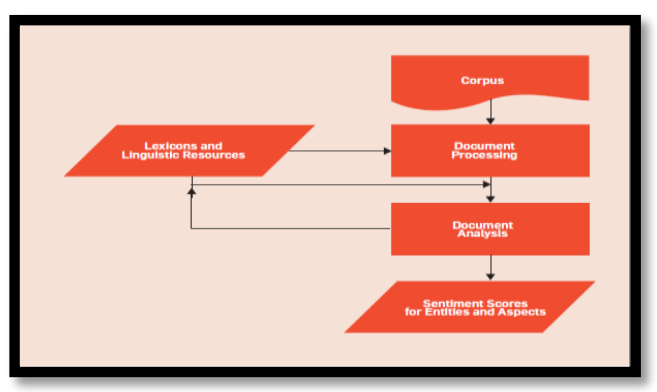

Figure 1: Architecture for generic sentimental analysis

The main component of the system is the document analysis module, which utilizes the linguistic resources to annotate the pre-processed documents with sentiment annotations. 
The annotations may be attached to whole documents (for document-based sentiment), to individual sentences (for sentence based sentiment) or to specific aspects of entities (for aspect-based sentiment). These annotations are the output of the system and they may be presented to the user using a variety of visualization tools [8].The paper is organized as follows. In section II-Literature review, In section III-Analysis and Discussion, In section IVConclusion.

\section{Literature review}

\section{II.1High level classification}

The task of Sentiment Analysis can be broadly classified into three levels: document level, sentence level, and feature based approaches (aspect level). [9]

\section{II.1.1Document level Sentiment Analysis}

Classification of the overall sentiment of a document is based on the overall sentiment of the opinion holder. This problem is basically a text classification problem. Here in general it is assumed that the document is written by a single person and expresses opinion about a single entity. One of the major challenges in the document level classification is that the entire sentence in a document may not be relevant in expressing the opinion about an entity. Therefore subjectivity/objectivity classification is very important in this type of classification. The irrelevant sentences must be eliminated from the processing works. Both supervised and unsupervised learning methods can be used for the document level classification. Any supervised learning algorithm like nave Bayes classifier, Support Vector Machine, or Maximum Entropy etc. can be used to train the system. For training and testing data, the reviewer rating (in the form of 1-5 stars), can be used. The features that can be used for the machine learning are term frequency, adjectives from Part of speech tagging, Opinion words and phrases, negations, dependencies etc. Labeling the polarities of the document manually is time consuming and hence the user rating available can be made use of. The unsupervised learning can be done by extracting the opinion words inside a document. The point-wise mutual information can be made use of to find the semantics of the extracted words. Thus the document level sentiment classification has its own advantages and disadvantages. Advantage is that we get an overall polarity of opinion text about a particular entity from a document. Disadvantage is that the different emotions about 
Journal of the ACS, Vol. 10, May 2019

different features of an entity could not be extracted separately. [11]

\section{II.1,2 Sentence level Sentiment Analysis}

Here polarity of each contributing sentence is derived. Again, here the assumption is that each sentence is written by a single person and expresses a single positive or negative opinion/sentiment. Sometimes Document-level sentiment classification is too coarse for our purpose. One of these reasons can be, the size of the document is too large or a more granular level of sentiments needs to be derived. A lot of early work in the region of sentence level analysis focuses on identifying subjective sentences. Most techniques use supervised learning. This can be divided into two tasks: first identify which sentence old opinion (subjective sentences) and then classify each sentence as positive/negative or the star rating. But there will be complex sentences also in the opinionated text. In such cases, sentence level sentiment classification is not desirable. Knowing that a sentence is positive or negative is of lesser use than knowing the polarity of a particular feature of a product. The advantage of sentence level analysis lies in the subjectivity/ objectivity classification. Some challenges in this approach could be: many objective sentences can imply sentiments or many subjective sentences do not express positive or negative sentiments/opinions. A single sentence may contain multiple opinions and subjective and factual clauses. [11]

\section{II.1.3 Feature based or Aspect level Sentiment Analysis}

It is a more granular approach that gives some extra information. For example; Sentiment classification at both the document and sentence (or clause) levels are useful, but they do not find what people liked and disliked. The product or the review they do not identify the targets of opinions. Much of the research is based on online reviews and blog related data, In the case of reviews, where the entity (product or service) is known. It's an easier problem. But for blogs, forum discussions, etc., it is much harder because the entity is unknown there may be many comparisons, and there is also a lot of irrelevant information. This problem is somewhat similar to the problem of Named Entity Resolution. Another interesting approach to solve sentiment analysis was presented in [10] by exploiting concept chains to build a graph model of a text. The model used Part of speech tagging to extract graph relations and performed graph based queries to get the overall sentiment of the text. [11] 
A survey on Sentimental Analysis algorithms and techniques

\section{II.2 Different categories, sub- approaches for Sentiment Classification techniques}

Sentiment Classification techniques can be roughly divided into machine learning approach, lexicon based approach and hybrid approach as shown in Table (1) [26].

Table (1)

\begin{tabular}{|c|c|c|c|}
\hline $\begin{array}{c}\text { Sentiment } \\
\text { approaches }\end{array}$ & classification & Features/techniques & $\begin{array}{c}\text { Advantages and } \\
\text { limitations }\end{array}$ \\
\hline $\begin{array}{l}\text { Machine } \\
\text { learning } \\
(\mathrm{ML})\end{array}$ & 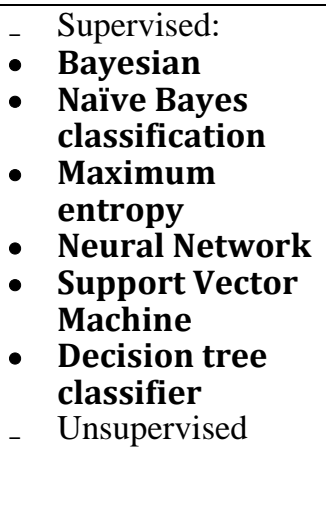 & $\begin{array}{ll}\text { - } & \text { Term presence } \\
\text { and frequency } \\
\text { Part of speech } \\
\text { information } \\
\text { Opinion words } \\
\text { and phrases }\end{array}$ & $\begin{array}{ll}\text { - } & \text { Advantages: } \\
\text { - } & \text { Create \& } \\
\text { adapt trained } \\
\text { models for } \\
\text { specific } \\
\text { proposes } \\
\text { - } \text { Limitations: } \\
\text { - } & \text { Irrelevance to } \\
\text { new data } \\
\text { because there } \\
\text { must be } \\
\text { labeled data } \\
\text { that could be } \\
\text { costly }\end{array}$ \\
\hline $\begin{array}{l}\text { Lexicon } \\
\text { based }\end{array}$ & $\begin{array}{l}\text { Dictionary Based } \\
\text { Corpus based } \\
\text { (Statistic or } \\
\text { semantic) }\end{array}$ & $\begin{array}{ll}\text { - } & \text { Dictionary } \\
\text { Based Corpus } \\
\text { based Manual }\end{array}$ & $\begin{array}{ll}\text { - } & \text { Advantage: } \\
\text { - } & \text { Cover a wider } \\
\text { set of words } \\
\text { - } & \text { Limitations: } \\
\text { - } & \text { Fixed } \\
\text { sentiment } \\
\text { orientation } \\
\text { and score of } \\
\text { words }\end{array}$ \\
\hline Hybrid & $\begin{array}{l}\text { Machine learning } \\
\text { lexicon based }\end{array}$ & $\begin{array}{ll}\text { Sentiment } \\
\text { lexicon } \\
\text { constructed } \\
\text { using public } \\
\text { resource } \\
\text { Sentiment } \\
\text { words as } \\
\text { features in ML } \\
\text { method }\end{array}$ & $\begin{array}{l}\text { - Advantage: } \\
\text { - Detection and } \\
\text { measurement } \\
\text { of sentiment } \\
\text { and its less } \\
\text { sensitive to } \\
\text { change in topic } \\
\text { domain } \\
\text { - Limitations: } \\
\text { - Noisy reviews }\end{array}$ \\
\hline
\end{tabular}




\section{II.2.1 Machine learning approach}

Machine learning approach relies on the famous ML algorithms to solve the SA as a regular text classification problem that makes use of syntactic and/or linguistic features.

Text Classification Problem Definition: We have a set of training records $D=\{X 1$, $\mathrm{X} 2, \mathrm{Xn}$ \} where each record is labeled to a class. The classification model is related to the features in the underlying record to one of the class labels. Then for a given instance of unknown class, the model is used to predict a class label for it. The hard classification problem is when only one label is assigned to an instance. The soft classification problem is when a probabilistic value of labels is assigned to an instance. [27]

\section{II.2.1.1Supervised learning}

The supervised learning methods depend on the existence of labeled training documents. There are many kinds of supervised classifiers in literature. In the next subsections, we present in brief details some of the most frequently used classifiers in SA. [27]

\section{1- Naive Bayes Classifier (NB)}

The Naive Bayes classifier is the simplest and most commonly used classifier. Naive Bayes classification model computes the posterior probability of a class, based on the distribution of the words in the document. The model works with the BOWs feature extraction which ignores the position of the word in the document. It uses Bayes Theorem to predict the probability that a given feature set belongs to a particular label as shown in equation (1).

$P($ label features $)=\frac{P(\text { label }) * P(\text { features } \mid \text { label })}{P(\text { features })}$

$\mathrm{P}$ (features) is the prior probability that a given feature set is occurred. Given the $\mathrm{Na} 1$ " ve assumption which states that all features are independent, the equation could be rewritten as shown in equation (2).

$P($ label features $)=\frac{P(\text { label }) * P(f 1 \mid \text { label }) * \ldots . . * P(\text { fn|label })}{P(\text { features })}$

An improved NB classifier was proposed by Kang and Yoo [12] to solve the problem of the tendency for the positive classification accuracy to appear up to approximately $10 \%$ higher than the negative classification accuracy. [27] 
A survey on Sentimental Analysis algorithms and techniques

\section{2- Maximum Entropy Classifier (ME).}

The Maxent Classifier (known as a conditional exponential classifier) converts labeled feature sets to vectors using encoding. This encoded vector is then used to calculate weights for each feature that can then be combined to determine the most likely label for a feature set. This classifier is parameterized by a set of $X$ \{weights\}, which is used to combine the joint features that are generated from a feature-set by an $X$ \{encoding\}. In particular, the encoding maps each $\mathrm{C}\{$ (featureset, label) $\}$ pair to a vector. The probability of each label is then computed using equation (3).

$P(f s \mid$ label $)=\frac{\operatorname{dotprod}(\text { weights, encode }(f s, l \text { label } l))}{\text { sum }(\operatorname{dotprod}(\text { weights, encode }(f s, l)) \text { forlinlabels })}$

ME classifier was used by Kaufmann [13] to detect parallel sentences between any language pairs with small amounts of training data.

\section{3- Support Vector Machines Classifiers (SVM).}

The main principle of SVMs is to determine linear separators in the search space which can best separate the different classes.

Text data are ideally suited for SVM classification becauseof the sparse nature of text, in which few features are irrelevant,but they tend to be correlated with one another andgenerally organized into linearly separable categories [15].

SVMs are used in many applications, among these applications are classifying reviews according to their quality. Chen and Tseng [16] have used two multiclass SVM- based approaches. [27]

SVMs were used by $\mathrm{Li}$ and $\mathrm{Li}$ [17] as a sentiment polarity classifier. Unlike the binary classification problem, they argued that opinion subjectivity and expresser credibility should also be taken into consideration. They proposed a framework that provides a compact numeric summarization of opinions on micro-blogs platforms. They identified and extracted the topics mentioned in the opinions associated with the queries of users, and then classified the opinions using SVM. They worked on Twitter posts for their experiment. They found out that the consideration of user credibility and opinion subjectivity is essential for aggregating micro-blog opinions. They proved that their mechanism can effectively discover market intelligence (MI) for 
supporting decision-makers by establishing a monitoring system to track external opinions on different aspects of a business in real time. [27]

\section{4- Neural Network (NN).}

Neural Network consists of many neurons where the neuron is its basic unit. The inputs to the neurons are denoted by the vector over line $\mathrm{Xi}$ which is the word frequencies in the $\mathrm{i}^{\text {th }}$ document. There are a set of weights $\mathrm{A}$ which are associated with each neuron used in order to compute a function of its inputs $\mathrm{f}\left(\_\right)$. The linear function of the neural network is: pi $1 / 4 \mathrm{~A} \_\mathrm{Xi}$. In a binary classification problem, it is assumed that the class label of Xi is denoted by yi and the sign of the predicted function pi yields the class label. [27]

Multilayer neural networks are used for non-linear boundaries. These multiple layers are used to induce multiple piecewise linear boundaries, which are used to approximate enclosed regions belonging to a particular class. The outputs of the neurons in the earlier layers feed into the neurons in the later layers. The training process is more complex because the errors need to be back-propagated over different layers. There are implementations of NNs for text data which are found in $[18,19]$.

\section{5- Decision tree classifiers.}

Decision tree classifier provides a hierarchical decomposition of the training data space in which a condition on the attribute value is used to divide the data [20]. The condition or predicate is the presence or absence of one or more words. The division of the data space is done recursively until the leaf nodes contain certain minimum numbers of records which are used for the purpose of classification. [27]

\section{II.2.1.2 The unsupervised approach}

The unsupervised approach was used too by Xianghua and Guo [23] to automatically discover the aspects discussed in Chinese social reviews and also the sentiments expressed in different aspects. They used LDA model to discover multi-aspect global topics of social reviews, then they extracted the local topic and associated sentiment based on a sliding window context over the review text. They worked on social reviews that were extracted from a blog data set (2000-SINA) and a lexicon (300-SINA Hownet). They showed that their approach obtained good topic partitioning results and helped to improve SA accuracy. It helped too to discover multi-aspect fine-grained topics and associated sentiment. [27] There are other unsupervised 
A survey on Sentimental Analysis algorithms and techniques

approaches that depend on semantic orientation using PMI [24] or lexical association using PMI, semantic spaces, and distributional similarity to measure the similarity between words and polarity prototypes [25].

\section{1- Lexicon-based approach}

Opinion words are employed in many sentiment classification tasks. Positive opinion words are used to express some desired states, while negative opinion words are used to express some undesired states. There are also opinion phrases and idioms which together are called opinion lexicon. There are three main approaches in order to compile or collect the opinion word list.

Manual approach is very time consuming and it is not used alone. It is usually combined with the other two automated approaches as a final check to avoid the mistakes that resulted from automated methods. The two automated approaches are presented in the following subsections. [27]

\section{2- Dictionary-based approach}

$[28,29]$ presented the main strategy of the dictionary-based approach. A small set of opinion words is collected manually with known orientations. Then, this set is grown by searching in the well-known corpora WordNet [30] or thesaurus [31] for their synonyms and antonyms. The newly found words are added to the seed list then the next iteration starts. The iterative process stops when no new words are found. After the process is completed, manual inspection can be carried out to remove or correct errors.

The dictionary based approach has a major disadvantage which is the inability to find opinion words with domain and context specific orientations. Qiu and He [34] used dictionary- based approach to identify sentiment sentences in contextual advertising. They proposed an advertising strategy to improve ad relevance and user experience. They used syntactic parsing and sentiment dictionary and proposed a rule based approach to tackle topic word extraction and consumers' attitude identification in advertising keyword extraction. [27]

\section{3- Corpus-based approach}

The Corpus-based approach helps to solve the problem of finding opinion words with context specific orientations. Its methods depend on syntactic patterns or patterns that occur together along with a seed list of opinion 
words to find other opinion words in a large corpus. One of these methods was represented by Hatzivassiloglou and McKeown [32].

The Conditional Random Fields (CRFs) method [33] was used as a sequence learning technique for extracting opinion expressions. It was used too by Jiaoa and Zhoua [23] in order to discriminate sentiment polarity by multistring pattern matching algorithm. Their algorithm was applied on Chinese online reviews. [27]

\section{Analysis and Discussion}

Table (2) summarize all proposed techniques, dataset used, problems that system solve, evaluation and limitation. [38, 42]

Table (2)

\begin{tabular}{|c|c|c|c|c|c|}
\hline Title & $\begin{array}{l}\text { Issues } \\
\text { Addressed }\end{array}$ & $\begin{array}{l}\text { Proposed } \\
\text { Technique }\end{array}$ & $\begin{array}{l}\text { Dataset } \\
\text { used }\end{array}$ & Accuracy & Limitations \\
\hline $\begin{array}{l}\text { Ke Wang and } \\
\text { Xiaojun Wan } \\
(2018) \\
{[38]}\end{array}$ & $\begin{array}{l}\text { predict } \\
\text { recommend } \\
\text { ations/decis } \\
\text { ions \& } \\
\text { polarity } \\
\text { shift }\end{array}$ & $\begin{array}{l}\text { Neural } \\
\text { network } \\
\text { model with a } \\
\text { novel abstract } \\
\text { based } \\
\text { attention } \\
\text { mechanism }\end{array}$ & $\begin{array}{l}\text { ICLR } \\
\text { (scholarl } \\
\text { y papers) }\end{array}$ & $\begin{array}{l}\text { Voting(3 } \\
\text { classes)=8 } \\
1.69 \\
\text { Confidenc } \\
\text { e based } \\
\text { average( } 3 \\
\text { classes)=6 } \\
9.02\end{array}$ & $\begin{array}{l}\text { Not suitable } \\
\text { for all } \\
\text { domains }\end{array}$ \\
\hline $\begin{array}{l}\text { Chen Y, } \\
\text { Zhang Z } \\
\text { (2018) [39] }\end{array}$ & $\begin{array}{l}\text { Improves } \\
\text { accuracy } \\
\text { text SA }\end{array}$ & $\begin{array}{l}\text { CNN } \\
\text { combined } \\
\text { with SVM }\end{array}$ & $\begin{array}{l}\text { NLPCC2 } \\
014\end{array}$ & $\begin{array}{l}\text { Precision( } \\
+)=0.89 \\
\text { Precision(- } \\
\text { )=0.886 }\end{array}$ & $\begin{array}{l}\text { Only for } \\
\text { Chinese }\end{array}$ \\
\hline $\begin{array}{l}\text { SM Shuhidan, } \\
\text { S R Hamidi et } \\
\text { al.(2018)[40] }\end{array}$ & $\begin{array}{l}\text { polarity } \\
\text { shift }\end{array}$ & $\begin{array}{l}\text { Opinion } \\
\text { lexicon based } \\
\text { and naïve } \\
\text { Bayes } \\
\text { algorithm }\end{array}$ & $\begin{array}{l}\text { Malaysia } \\
\text { financial } \\
\text { headlines } \\
\text { news }\end{array}$ & $\begin{array}{l}\text { No } \\
\text { accuracy } \\
\text { mentioned }\end{array}$ & \\
\hline $\begin{array}{l}\text { I. El Alaoui, } \\
\text { Y. Gahi,et } \\
\text { al. }(2018)[41]\end{array}$ & $\begin{array}{l}\text { polarity } \\
\text { shift }\end{array}$ & $\begin{array}{l}\text { lexicon based } \\
\text { approach is an } \\
\text { unsupervised } \\
\text { learning } \\
\text { method }\end{array}$ & $\begin{array}{l}\text { Tweet(20 } \\
16 \text { US } \\
\text { election) }\end{array}$ & $\begin{array}{l}\text { Mean } \\
\text { accuracy= } \\
90 \%\end{array}$ & $\begin{array}{l}\text { Limited to } \\
\text { twitter data }\end{array}$ \\
\hline $\begin{array}{l}\text { Han, Y., Liu, } \\
\text { Y., Jin, } \\
\text { Z(2019)[42] }\end{array}$ & $\begin{array}{l}\text { improving } \\
\text { the } \\
\text { accuracy by } \\
\text { extending } \\
\text { the labeled } \\
\text { data }\end{array}$ & $\begin{array}{l}\text { semi- } \\
\text { supervised } \\
\text { model based } \\
\text { on dynamic } \\
\text { threshold and } \\
\text { multi- } \\
\text { classifier }\end{array}$ & $\begin{array}{l}\text { Large } \\
\text { Movie } \\
\text { Review } \\
\text { Dataset } \\
\text { v1.0 }\end{array}$ & $\begin{array}{l}\mathrm{F} \\
\text { measure(1 } \\
000 \\
\text { labeled } \\
\text { training } \\
\text { data } \\
\text { number)= } \\
82.69\end{array}$ & $\begin{array}{l}\text { Limited } \\
\text { base learner }\end{array}$ \\
\hline
\end{tabular}




\section{Conclusion}

Sentiment analysis (or) opinion mining plays a significant role in business decision making. Many of the organizations and enterprises will take their business decision only based on their customer review. There are several techniques for performing sentiment analysis. After going through all of these papers, there were no interest in other language other than English and Chinese cause of lack of resource and researches concerning other languages, A future challenge in applying sentimental analysis on social media data and apply deep learning algorithm on aspect based more and working more on generating dynamic labeled data to improve the performance.

\section{References}

[1] Banea, C., Mihalcea, R., and Wiebe, J., "Multilingual Sentiment and Subjectivity Analysis", In Multilingual Natural Language Processing”, editors Imed Zitouni and Dan Bikel, Prentice Hall, 2011.

[2] Hassena, R.P., "Challenges and Applications", International Journal of Application or Innovation in Engineering \& Management (IJAIEM), Volume 3, Issue 5,2014

[3] Richard, S., Alex, P., Jean, Y.W., Jason, C., Christopher, D.M., Andrew, Y.N. and Christopher, P., "Recursive Deep Models for Semantic Compositionality Over a Sentiment Treebank", Conference on Empirical Methods in Natural Language Processing, 2013.

[4] Choudhari, and Rajankar, Introduction to Natural Language Processing With Python, International Journal of Modern Trends in Engineering and Research (IJMTER)Volume 02, Issue 04, [April - 2015].

[5] A. Montoyo, P. Martínez-Barco, A. Balahur, Subjectivity and sentiment analysis: An overview of the current state of the area and envisaged developments, Decision Support Systems 53 (2012) 675-679.

[6] S. Poria, A. Gelbukh, A. Hussain, N. Howard, D. Das, S. Bandyopadhyay, Enhanced SenticNet with Affective Labels for Concept- Based Opinion Mining, KnowledgeBased Approaches to Concept-Level Sentiment Analysis, IEEE Intelligent Systems, (2013) 1.

[7] B. Liu, Sentiment analysis: A multi-faceted problem, IEEE Intelligent Systems 25, no. 3 (2010): 76-80.

[8] "Techniques and applications for sentiment analysis" Ronen Feldman Communications of the ACM - 2013 
[9] Bing Liu, "Sentiment Analysis and Subjectivity"

[10] Wei jin, Srihari, "Mining Hidden Associations in Text Corpora through Concept Chain and Graph Queries“"

[11] Rahul Tejwani, "Sentiment Analysis: A Survey"

[12] Kang Hanhoon, Yoo Seong Joon, Han Dongil. Senti-lexicon and improved Nai"ve Bayes algorithms for sentiment analysis of restaurant reviews. Expert Syst Appl 2012; 39:6000-10.

[13] Kaufmann JM. JMaxAlign: A Maximum Entropy Parallel Sentence Alignment Tool. In: Proceedings of COLING'12: Demonstration Papers, Mumbai; 2012. p. 277-88.

[14] Vapnik V. The nature of statistical learning theory, New York; 1995.

[15] Joachims T. Probabilistic analysis of the rocchio algorithm with TFIDF for text categorization. In: Presented at the ICML conference; 1997.

[16] Cortes C, Vapnik V. Support-vector networks, presented at the Machine Learning; 1995.

[17] Chin Chen Chien, Tseng You-De. Quality evaluation of product reviews using an information quality framework. Decis Support Syst 2011; 50:755-68.

[18] Li Yung-Ming, Li Tsung-Ying. Deriving market intelligence from microblogs. Decis Support Syst 2013.

[19] Ruiz M, Srinivasan P. Hierarchical neural networks for text categorization. In: Presented at the ACM SIGIR conference; 1999.

[20] Ng Hwee Tou, Goh Wei, Low Kok. Feature selection, perceptron learning, and a usability case study for text categorization. In: Presented at the ACM SIGIR conference; 1997.

[21] Quinlan JR. Induction of decision trees. Machine Learn 1986; 1:81-106.

[22] Liu Bing, Hsu Wynne, Ma Yiming. Integrating classification and association rule mining. In: Presented at the ACM KDD conference; 1998.

[23] Medhat W, Hassan A, Korashy H. Combined algorithm for data mining using association rules. Ain Shams J Electric Eng 2008; 1(1).

[24] Xianghua Fu, Guo Liu, Yanyan Guo, Zhiqiang Wang. Multiaspect sentiment analysis for Chinese online social reviews based on topic modeling and HowNet lexicon. Knowl-Based Syst2013; 37:186-95.

[25] Turney P. Thumbs up or thumbs down? semantic orientation applied to unsupervised classification of reviews. In: Proceedings of annual meeting of the Association for Computational Linguistics (ACL'02); 2002.

[26] Read J, Carroll J. Weakly supervised techniques for domainindependent sentiment classification. In: Proceeding of the 1st international CIKM workshop on topicsentiment analysis for mass opinion; 2009. p. 45-52.

[27] Diana Maynard, Adam Funk. Automatic detection of political opinions in tweets. In: Proceedings of the 8th international conference on the semantic web, ESWC'11; 2011. p. 88-99.

[28] Sentiment analysis algorithms and applications: A survey Walaa Medhat-Ahmed Hassan-Hoda Korashy - Ain Shams Engineering Journal - 2014

[29] Hu Minging, Liu Bing. Mining and summarizing customer reviews. In: Proceedings of ACM SIGKDD international conference on Knowledge Discovery and Data Mining (KDD'04); 2004. 
A survey on Sentimental Analysis algorithms and techniques

[30] Kim S, Hovy E. Determining the sentiment of opinions. In: Proceedings of interntional conference on Computational Linguistics (COLING'04); 2004.

[31] Miller G, Beckwith R, Fellbaum C, Gross D, Miller K. WordNet: an on-line lexical database. Oxford Univ. Press; 1990.

[32] Mohammad S, Dunne C, Dorr B. Generating high-coverage semantic orientation lexicons from overly marked words and a thesaurus. In: Proceedings of the conference on Empirical Methods in Natural Language Processing (EMNLP'09); 2009.

[33] Hatzivassiloglou V, McKeown K. Predicting the semantic orientation of adjectives. In: Proceedings of annual meeting of the Association for Computational Linguistics (ACL'97); 1997.

[34] Lafferty J, McCallum A, Pereira F. Conditional random fields: probabilistic models for segmenting and labeling sequence data.In: Proceedings of International Conference on Machine Learning (ICML'01); 2001.

[35] Qiu Guang, He Xiaofei, Zhang Feng, Shi Yuan, Bu Jiajun, Chen Chun. DASA: dissatisfaction-oriented advertising based on sentiment analysis. Expert Syst Appl 2010;37:6182-91.

[36] Farman Alia, Kyung-Sup Kwaa,Yong-Gi Kimb,” Opinion mining based on fuzzy domain ontology and Support Vector Machine: A proposal to automate online review classification", Applied Soft Computing-2016.

[37] Approaches, Tools and Applications for Sentiment Analysis Implementation Alessia D'andrea-Fernando Ferri-Patrizia Grifoni-Tiziana Guzzo - International Journal of Computer Applications - 2015

[38] Ke Wang and Xiaojun Wan. Sentiment analysis of peer review texts for scholarly papers. In The 41st International ACM SIGIR Conference on Research \& Development in Information Retrieval, SIGIR 2018, Ann Arbor, MI, USA, July 08-12, 2018, pp. 175-184, 2018b. Doi: 10.1145/3209978. 3210056.

[39] Chen Y, Zhang Z (2018) Research on text sentiment analysis based on CNNs and SVM. In: 13th IEEE conference on industrial electronics and applications (ICIEA), pp. 2731-2734

[40] S M Shuhidan, S R Hamidi, S Kazemian et al., "Sentiment Analysis for Financial News Headlines using Machine Learning Algorithm[M]", Proceedings of the 7th International Conference on Kansei Engineering and Emotion Research, 2018.

[41] I. El Alaoui, Y. Gahi, R. Messoussi, Y. Chaabi, A. Todoskoff, A. Kobi, "A novel adaptable approach for sentiment analysis on big social data", Journal of Big Data, vol. 5, no. 1, 2018.

[42] Han, Y., Liu, Y., Jin, Z "Sentiment analysis via semi-supervised learning: a model based on dynamic threshold and multi-classifiers", Journal Neural Computing and Applications, pp1-13 (2019). https://doi.org/10.1007/s00521-018-3958-3 